\title{
Two-step emulsion PCR to prevent formation of the chimeric molecules
}

E. Omelina ${ }^{1 *}$, A. Ivankin ${ }^{1}$, A. Leshchenko ${ }^{1,2}$, L. Yarinich ${ }^{1}$, M. Lebedev ${ }^{1,2}$,

A. Pindyurin ${ }^{1}$

${ }^{1}$ Institute of Molecular and Cellular Biology SB RAS, Novosibirsk, Russia

${ }^{2}$ Novosibirsk State University, Novosibirsk, Russia

*e-mail:omelina@mcb.nsc.ru

Key words: emulsion PCR, chimeric molecules, next-generation sequencing

Motivation and Aim: Application of the conventional PCR method for the amplification of the random nucleotide sequence libraries often causes a formation of the undesired chimeric molecules. Invention of the water-in-oil emulsion PCR (ePCR) approach allowed to reduce the probability of the chimeric molecule formation compared to the conventional PCR. However, in the non-optimized conditions even ePCR causes the formation of the chimeric products.

Methods and Algorithms: We developed a step-by-step protocol for the ePCR consisting of two consequent rounds. We found that both an initial amount of the DNA template and number of amplification cycles play a critical role in the formation of the chimeric molecules. We suggest to use only $10^{6}$ DNA molecule copies for the first round of ePCR. Results: We analyzed a formation of the chimeric products during amplification of heterogeneous plasmid library in different conditions. To assess the percent of the formed chimers we used Illumina MiSeq platform. A proportion of chimeric molecules under optimal conditions was lower than $0.25 \%$. We suppose that this two-step ePCR approach may be useful for the preparation of heterogeneous sequence libraries for the next-generation sequencing and other issues which demand avoiding of the chimeric molecule formation.

Conclusion: ePCR approach allows to separate the DNA template molecules from each other using the water-in-oil emulsion. Our data suggest that ePCR approach is suitable for the preparation of random DNA libraries for the next-generation sequencing. However it requires additional adjustment to reduce a formation of chimeric molecules as much as possible.

Acknowledgements: Supported by the RSF (16-14-10288). 